\title{
IMMERSED SURFACES AND PENCILS OF PLANES IN 3-SPACE
}

\author{
by F. J. CRAVEIRO dE CARVALHO
}

(Received 7 April, 1979)

1. Introduction. Let $M$ be a compact connected boundaryless surface and $f: M \rightarrow$ $\mathbb{R}^{3}$ a smooth immersion transverse to a straight line $L$. Thus there is an even number $p$ of points $x \in M$ such that $f(x) \in L$. Under further transversality assumptions on $f$ (see $\$ 3$ ) there is a finite number $q$ of points $x$ of $M$ such that the plane containing $f(x)$ and $L$ touches $f(M)$ at $f(x)$. These assumptions are mild in the sense that they hold for any $f$ in an open dense subset of the space of smooth immersions under consideration. Suppose that the Gaussian curvature of $f(M)$ is positive at $q^{+}$of these points and negative at $q^{-}$, with $q=q^{+}+q^{-}$. Then

$$
p+q^{+}-q^{-}=e(M)
$$

where $e(M)$ denotes the Euler number of $M$.

The proof is an application of the Poincaré-Hopf theorem (see [1], [2]). Our theorem may be interpreted as a development of the theory of horizon immersions for surfaces. In particular the main result of [3] is a consequence of our result.

We illustrate the relation $(*)$ with a few examples. Take the standard embedding of the torus $T$ into $\mathbb{R}^{3}$, and let $L$ be parallel to the axis of $T$. In Figure 1, we show three positions of $L$ viewed from above.

I am grateful to Stewart Robertson for suggesting this problem and I thank him and David Chillingworth for many helpful discussions.

2. Notations. In what follows we shall be dealing with compact connected smooth $\left(=C^{\infty}\right)$ boundaryless manifolds. All the maps are smooth unless otherwise stated.

Given manifolds $M$ and $N$ and a map $g: M \rightarrow N$ the derivative of $g$ is denoted by $g_{*}: T M \rightarrow T N$, and $g_{* x}: T_{\mathrm{x}} M \rightarrow T_{\mathrm{g}(\mathrm{x})} N$ denotes the restriction of $g_{*}$ to the tangent space to $M$ at $x$. The critical set of $g$ is denoted by $C(\mathrm{~g})$.

If $f: M \rightarrow \mathbb{R}^{n+1}$ (where $\operatorname{dim} M=n$ ) is an immersion, then we denote by $T_{x}$ the affine tangent $n$-plane to $f(M)$ at $f(x)$. Such an immersion induces a map $F: M \rightarrow \mathbb{R}_{n}^{n+1}$, where $\mathbb{R}_{n}^{n+1}$ denotes the Grassmannian of affine $n$-planes in $\mathbb{R}^{n+1}$. By the Gaussian curvature of $f(M)$ at $f(m)$ we mean the Gaussian curvature of $f(U)$ at $f(m)$, where $U$ is an oriented open neighbourhood of $m$ and $f \mid U$ is an embedding.

We may assume that the line $L$ in $\mathbb{R}^{3}$ is defined by $x_{1}=x_{2}=0$. The pencil $\tilde{L}$ of 2-planes containing $L$, as a submanifold of $\mathbb{R}_{2}^{3}$, is diffeomorphic to the 1-dimensional real projective space $P^{1}$. For $P^{1}$ we shall consider the two standard charts $\psi: V \rightarrow \mathbb{R}$, $\psi^{\prime}: V^{\prime} \rightarrow \mathbb{R}$, where

$$
V\left(\text { resp. } V^{\prime}\right)=\left\{\pi \varepsilon P^{1} \mid \pi \text { is defined by }(1, x)(\text { resp. }(x, 1))\right\}
$$

and $\psi(\pi)=x, \psi^{\prime}(\pi)=x$.

Glasgow Math. J. 22 (1981) 133-136. 


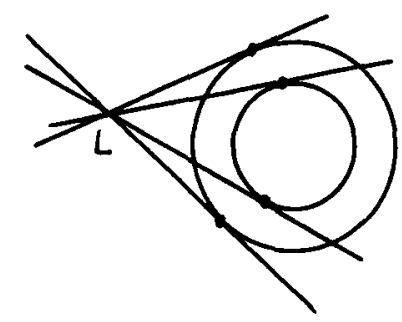

$p=0, q^{+}=q^{-}=2$

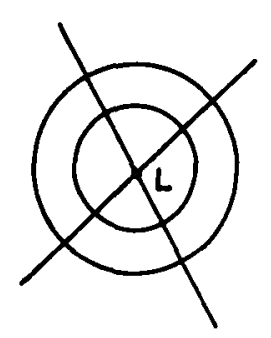

$p=q^{+}=q^{-}=0$

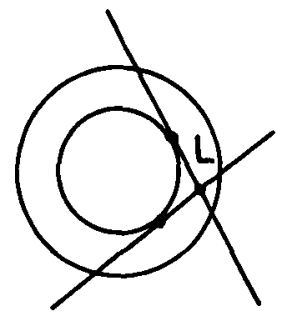

$p=q^{-}=2, q^{+}=0$

Figure 1

For any immersion $f: M \rightarrow \mathbb{R}^{3}$, with $\operatorname{dim} M=2$, we shall use three types of chart $\alpha, \beta, \gamma$ for $M$ where

$$
\begin{aligned}
& f \circ \alpha^{-1}(x, y)=(x, y, g(x, y)), \\
& f \circ \beta^{-1}(x, y)=(x, h(x, y), y), \\
& f \circ \gamma^{-1}(x, y)=(k(x, y), x, y)
\end{aligned}
$$

Finally $I_{2}(F, \tilde{L})$ will denote the intersection number $\bmod (2)$ of $F$ with $\tilde{L}$.

3. The main result. The proof of the relation stated in $\$ 1$ will occupy the whole of this section. We start with two elementary observations.

3.1. Suppose $f^{-1}(L) \neq \varnothing$. Then $f \Uparrow L$ at $m \in f^{-1}(L)$ iff $T_{m}$ intersects $L$ in a point.

3.2. Suppose $F^{-1}(\tilde{L}) \neq \varnothing$. Then $F \nwarrow \tilde{L}$ at $m \in F^{-1}(\tilde{L})$ iff

(a) $f(m) \notin L$,

(b) $f(M)$ has non-zero Gaussian curvature at $f(m)$.

Bearing in mind 3.2, we suppose, from now on, that $F \nwarrow \tilde{L}$. Thus the condition $f \nwarrow L$ follows automatically (see $\$ 1$ ).

Let $\Delta=f^{-1}(L)$. Define a map $\phi: M \backslash \Delta \rightarrow P^{1}$ by associating to $m \in M \backslash \Delta$ the 2-plane containing $f(m)$ and $L$. The map $\phi$ is smooth and $m \in C(\phi)$ iff $T_{m} \in \tilde{L}$. Since $F \nwarrow \tilde{L}$, the set $C(\phi) \cup \Delta$ is finite.

Consider the vector fields $\operatorname{grad}\left(\psi^{\circ} \phi\right)$ and $\operatorname{grad}\left(\psi^{\prime} \circ \phi\right)$. We use these to obtain a welldefined vector field $Z$ on $M \backslash\{\Delta \cup C(\phi)\}$ as follows:

$$
Z(m)=\left\{\begin{array}{lll}
\operatorname{grad}(\psi \circ \phi)(m) /\|\operatorname{grad}(\psi \circ \phi)(m)\| & \text { if } & m \in \phi^{-1}(V), \\
-\operatorname{grad}\left(\psi^{\prime} \circ \phi\right)(m) /\left\|\operatorname{grad}\left(\psi^{\prime} \circ \phi\right)(m)\right\| & \text { if } & m \in \phi^{-1}\left(V^{\prime}\right)
\end{array}\right.
$$

Take a smooth function $\gamma^{\prime}: M \rightarrow \mathbb{R}$, such that

$$
\begin{array}{lll}
\gamma^{\prime}(x)=0 & \text { if } & x \in \Delta \cup C(\phi), \\
\gamma^{\prime}(x)>0 & \text { if } & x \in M \backslash\{\Delta \cup C(\phi)\} .
\end{array}
$$


Using $\gamma^{\prime}$ we define another vector field $Y$ by

$$
Y(m)=\left\{\begin{array}{lll}
\gamma^{\prime}(m) Z(m) & \text { if } & m \in M \backslash\{\Delta \cup C(\phi)\}, \\
0 & \text { if } & m \in \Delta \cup C(\phi)
\end{array}\right.
$$

It is not hard to show that $Y$ is continuous. If $\phi(m)$ is a regular value of $\phi$, then $Y(m)$ is orthogonal to the contour of $\phi$ passing through $m$.

As we see, we are now in a position which allows us to use the Poincaré-Hopf theorem. To calculate the indices at the zeros of $Y$ we shall distinguish two cases:

(a) $m_{0}$ is a zero of $Y$ and $m_{0} \in C(\phi)$;

(b) $m_{0}$ is a zero of $Y$ and $m_{0} \in \Delta$.

Case (a). Due to the transversality condition we have imposed, both $\psi \circ \phi$ and $\psi^{\prime} \circ \phi$ are Morse functions. Then the index of $m_{0}$ as a zero of $Y$ can be calculated by looking at the index of $m_{0}$ as a critical point of either $\psi \circ \phi$ or $-\left(\psi^{\prime} \circ \phi\right)$. It follows from standard calculations that the index is 1 or -1 according as the Gaussian curvature at $f\left(m_{0}\right)$ is positive or negative.

Case (b). Let $m_{0} \in \Delta$. As $f\left(m_{0}\right) \in L$ and $f \AA L$ it is possible to find a chart $\alpha: U \rightarrow U^{\prime}$ such that $f \mid U$ is an embedding, $f \circ \alpha^{-1}(x, y)=(x, y, g(x, y))$ and no other point of $\Delta \cup C(\phi)$, other than $m_{0}$, is in $U$. The restriction $f \mid U$ is transversal to any $\pi \in \tilde{L}$. For any $m \in U \backslash\left\{m_{0}\right\}$, we have that $Y(m)$ is orthogonal to the contour of $\phi \mid U \backslash\left\{m_{0}\right\}$ through $m$, and this contour is $(f \mid U)^{-1}(\pi) \backslash\left\{m_{0}\right\}$, where $\phi(m)=\pi$. Moreover $(f \mid U)^{-1}(\pi)$ is a 1dimensional submanifold of $M$.

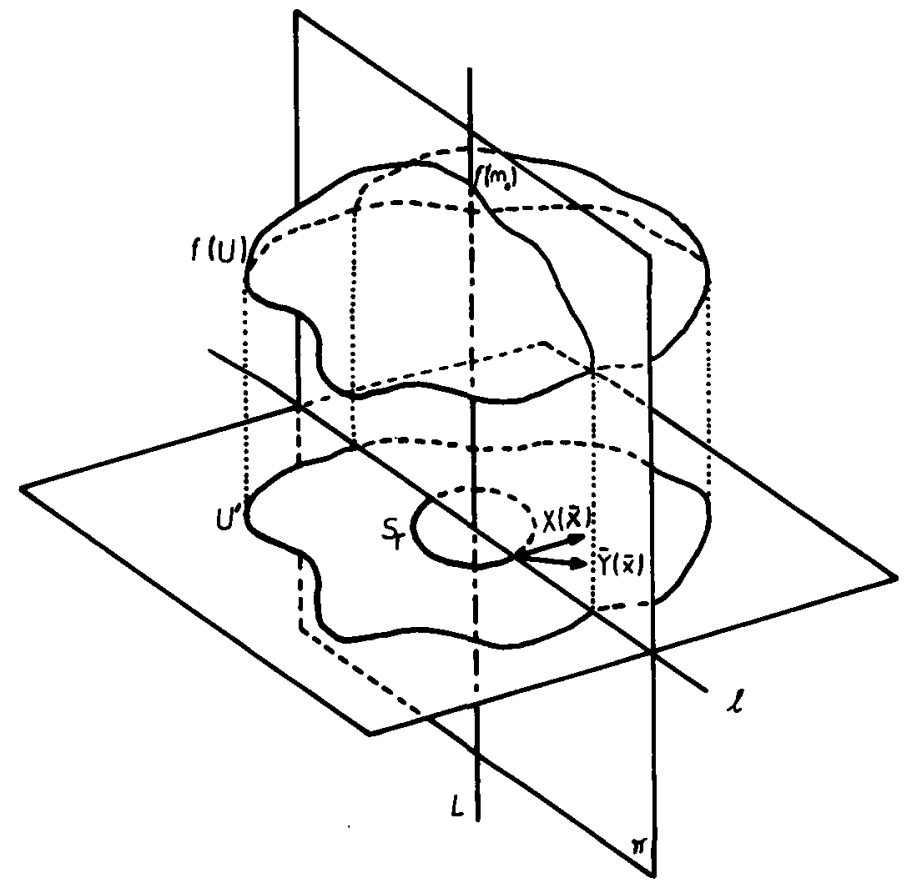

Figure 2 
If $\pi$ is as shown in Figure 2, $(f \mid U)^{-1}(\pi)$ is diffeomorphic to $U^{\prime} \cap l$, where $l=$ $\left(\mathbb{R}^{2} \times\{0\}\right) \cap \pi$. Using the chart $\alpha$ we get the "pullback" $\bar{Y}$ of $Y$, defined on $U^{\prime}$. This vector field is never zero except at the origin. The important remark to make about $\bar{Y}$ is that because $Y(m)$ is orthogonal to $i_{* m}\left(T_{m}\left((f \mid U)^{-1}(\pi)\right)\right)$ then

$$
\bar{Y}(\bar{x}) \notin i_{* \bar{x}}\left(T_{\bar{x}}\left(U^{\prime} \cap l\right)\right) \text {, }
$$

for any $m$ such that $\alpha(m)=\bar{x} \neq(0,0)$. Here we have denoted the obvious inclusions by $i$, and $\bar{x}=(x, y)$.

Let $S_{\mathrm{r}}$ be a small sphere round the origin such that $S_{\mathrm{r}} \subset U^{\prime}$. We define a vector field $X$ of unit norm on $S_{r}$ and tangent to $S_{r}$ such that at a particular point $\bar{x}, X(\bar{x})$ and $\bar{Y}(\bar{x})$ form an acute angle. Looking at the vector fields as maps into $\mathbb{R}^{2}$,

$$
X: S_{r} \rightarrow S^{1} \subset \mathbb{R}^{2}, \quad \bar{Y} \mid S_{r}: S_{r} \rightarrow \mathbb{R}^{2},
$$

we see that they are homotopic, a homotopy $H: S_{r} \times[0,1] \rightarrow \mathbb{R}^{2}$ being defined by

$$
H(\bar{x}, t)=t X(\bar{x})+(1-t) \bar{Y}(\bar{x}) .
$$

The map $H$ is never zero and therefore induces a homotopy between $X$ and $\bar{Y}_{1}: S_{r} \rightarrow S^{1}$, where $\bar{Y}_{1}(\bar{x})=\bar{Y}(\bar{x}) /\|\bar{Y}(\bar{x})\|$. Consequently the maps $X$ and $\bar{Y}_{1}$ have the same degree and the degree of $X$ is 1 . Hence the index of $Y$ at $m_{0}$ is 1 .

Having calculated the indices, we can deduce the relation $(*)$ in $\S 1$ immediately.

4. Consequences of the main result. The theorem we have just proved yields the following two corollaries.

Corollary 4.1. Assume F木 $\tilde{L}$. Then $I_{2}(F, \tilde{L}) \equiv e(M)(\bmod 2)$.

Proof. By intersection theory, $I_{2}(f, L)=0$. The result follows now from $(*)$ in $\$ 1$.

The next corollary is just the theorem on the existence of horizon maps for surfaces [3].

COROLlaRY 4.2. If $f$ is a horizon immersion then $M$ is diffeomorphic to $S^{2}, S^{1} \times S^{1}$ or the Klein bottle. If $M$ is diffeomorphic to $S^{2}$ then $\# f^{-1}(L)=2$; otherwise, $\# f^{-1}(L)=0$.

Proof. If $f$ is a horizon immersion then $F 木 \tilde{L}$ and $F^{-1}(\tilde{L})=\varnothing$. Because $\# f^{-1}(L)$ is even it follows from $(*)$ in $\S 1$ that $e(M)$ is even and greater than or equal to zero.

This paper was prepared while the author held a scholarship from INIC-LISBON.

\section{REFERENCES}

1. P. Alexandroff and H. Hopf, Topologie I (Springer, 1935).

2. J. Milnor, Topology from the differentiable viewpoint (The University of Virginia Press 1965).

3. S. A. Robertson, The dual of a height function, J. London Math. Soc. (2) 8 (1974), 187-192.

Departamento de Matemática

UNIVERSIDADE DE COIMBRA

Portugal 